\title{
RESPOSTA DE MUDAS DE TUCUMÃ À APLICAÇÃO DE DOSES DE CALCÁRIO E FÓSFORO
}

Vinícius Ide Franzini ${ }^{1}$, Alysson Roberto Baizi e Silva ${ }^{2}$, Daniel da Silva Tavares ${ }^{3}$, Juliane Batista Maciel ${ }^{3}$

1 Pesquisador da Embrapa Amazônia Oriental, Laboratório de Solos, Belém, Pará, Brasil (vinicius.franzini@embrapa.br)

2 Pesquisador da Embrapa Amazônia Oriental, Laboratório de Solos, Belém, Pará, Brasil

3 Aluno de graduação de Agronomia da Universidade Federal Rural da Amazônia (UFRA), Belém, Pará, Brasil

Recebido em: 08/04/2016 - Aprovado em: 30/05/2016 - Publicado em: 20/06/2016 DOI: 10.18677/Enciclopedia_Biosfera_2016_103

\begin{abstract}
RESUMO
A demanda por óleos vegetais tem aumentado no mundo. O tucumã (Astrocaryum vulgare Mart), que é uma palmeira nativa da Amazônia, é uma alternativa para a produção de óleo. No Pará, a camada superficial do solo, que é comumente utilizada como substrato para a formação de mudas, geralmente apresenta acidez elevada e baixa disponibilidade de fósforo $(\mathrm{P})$. Diante do exposto, objetivou-se estimar o efeito da calagem e da adubação fosfatada no desenvolvimento de mudas de tucumã. $O$ delineamento experimental foi o inteiramente casualizado, com seis repetições, em esquema fatorial $4 \times 6$, correspondendo a quatro níveis de saturação por bases $(\mathrm{V})$ $(13,40,65$ e $90 \%)$ e seis doses de $P\left(0,30,60,120,240\right.$ e $\left.480 \mathrm{mg} \mathrm{dm}^{-3}\right)$. O experimento foi desenvolvido em viveiro da empresa Dentauá, em Santo Antônio do Tauá, Pará. As plantas foram cultivadas por 350 dias, em sacos de polietileno preto e perfurado, contendo $3,0 \mathrm{dm}^{-3}$ de substrato. Observou-se interação entre níveis de $V$ e doses de $P$ apenas para a massa de matéria seca de parte aérea, de raiz e total, que aumentaram em resposta à aplicação de doses de calcário e fertilizante fosfatado. A calagem e a adubação fosfatada aumentaram o diâmetro do coleto e número total de folhas, mas não influenciaram a altura da planta e o índice SPAD. A correção da acidez do solo e o fornecimento de P melhoraram o desenvolvimento de mudas de tucumã.
\end{abstract}

PALAVRAS-CHAVE: calagem, fertilizante fosfatado, solos ácidos

\section{TUCUMÃ SEEDLINGS RESPONSE TO THE APPLICATION OF LIMESTONE AND PHOSPHORUS RATES}

\begin{abstract}
The demand for vegetable oils has increased around the world. Tucumã palm (Astrocaryum vulgare Mart), which is native from Amazon, is an alternative crop for the production of oil. In Pará state, topsoil is usually used as substrate for the production of seedlings in nursery, but it is acidic and has low availability of phosphorus $(P)$. The objective of this study was to evaluate the effect of liming and $P$ fertilization on growth of tucumã seedlings. The experiment was arranged in a
\end{abstract}


completely randomized design, in a $4 \times 6$ factorial scheme with six replications. The treatments consisted of four levels of base saturation (V) (13, 40, 65 e $90 \%)$ and six $P$ rates $\left(0,30,60,120,240\right.$ e $\left.480 \mathrm{mg} \mathrm{dm}^{-3}\right)$. The experiment was conducted at a nursery of the Company Dentauá, municipality of Santo Antônio do Tauá, state of Pará. Seedlings were grown during 350 days, in black holed polyethylene bags filled with $3.0 \mathrm{dm}^{-3}$ of soil. Significant interactions between $V$ levels and $P$ rates were observed only for shoot, root and total dry matter mass. The application of limestone and $P$ fertilizer significantly increased shoot, root and total dry matter, collar diameter and number of leaves per plant, but it did not influence plant height and SPAD index. Liming and $P$ fertilization improved growth of tucumã seedlings.

KEYWORDS: liming, phosphorus fertilizer, acid soils

\section{INTRODUÇÃO}

Cada vez se torna mais importante aumentar a produção de óleos vegetais para suprir as demandas da população. O consumo mundial de óleo vegetal mais que dobrou nos últimos 20 anos (FAO, 1995; USDA, 2015). Em 2015, a produção mundial de óleo vegetal foi de aproximadamente 180 milhões de toneladas (USDA, 2016) e, estima-se que, em 2050, serão necessários 240 milhões de toneladas de óleo vegetal comestível para suprir as necessidades da população (CORLEY, 2009; RIVAL \& LEVANG, 2014). Além disso, o aumento da demanda mundial por fontes renováveis de energia, como o biodiesel, por causa de seus benefícios econômicos e ambientais, também aumentará a demanda por óleos vegetais (NO, 2011; ATABANI et al., 2013). A demanda estimada por óleos vegetais para a produção de biodiesel é de 330 milhões de toneladas em 2050, superando a do mercado de alimentos (KOH, 2007; CORLEY, 2009).

O mercado mundial de óleo vegetal é composto principalmente por produtos obtidos em quatro oleaginosas (palma de óleo, soja, colza e girassol), sendo que os óleos de palma e de soja correspondem a mais de $60 \%$ do total produzido (USDA, 2013). No Brasil, a principal fonte de óleo vegetal é a soja (OSAKI \& BATALHA, 2011). Alternativamente a essas culturas, na região Amazônica encontra-se o tucumã (Astrocaryum vulgare Mart), que é uma espécie nativa. Trata-se de uma palmeira pertencente à família Arecaceae, cujos frutos possuem alto teor de vitamina A e, além de serem utilizados para extração de óleo do mesocarpo e da amêndoa, também são consumidos in natura e usados como matéria-prima pela indústria alimentícia (SHANLEY et al., 2005; NASCIMENTO \& OLIVEIRA, 2011).

A utilização de mudas vigorosas, principalmente em culturas perenes, aumenta a chance de sucesso na implantação, a sobrevivência das mudas no campo, o desenvolvimento das plantas e a longevidade da cultura, a produção e qualidade do produto e potencializa o nível de resposta a tecnologia empregada na plantação (SILVA et al., 2000; OLIVEIRA et al., 2004; TUCCI et al., 2009; OLIVEIRA et al., 2012). Em algumas culturas, estima-se que $60 \%$ do sucesso da plantação está em implantá-la com mudas de qualidade (ZACCHEO et al., 2013).

A qualidade das mudas está diretamente relacionada ao seu estado nutricional, que é afetado pela fertilidade do substrato (NATALE et al., 2004; TUCCI et al., 2009; VALENCIA et al., 2010). As limitações da fertilidade do substrato é um dos fatores responsáveis por perdas de mudas e pela elevada mortalidade de plantas após 0 plantio definitivo no campo (TUCCl et al., 2009).

$A$ acidez do solo e a deficiência de $P$ estão entre as principais causas da baixa produtividade agrícola em regiões tropicais no mundo, como no Brasil (FAGERIA \& NASCENTE, 2014; RAIJ, 2011). O uso de terra do subsolo para a produção de 
mudas em viveiro possui a vantagem de potencialmente reduzir a ocorrência de doenças, mas o subsolo, geralmente, apresenta acidez elevada e baixa disponibilidade de nutrientes (TUCCI et al., 2009). No estado do Pará, a camada superficial do solo, que é comumente utilizada como substrato para a produção de mudas, geralmente, também apresenta acidez elevada e baixa disponibilidade de fósforo $(\mathrm{P})$. Os solos da região Amazônica, de modo geral, são caracterizados pelo grau alto de intemperismo, acidez elevada, baixa disponibilidade de nutrientes e capacidade alta de adsorção de P (SANCHEZ \& COCHRANE, 1980; FALCÃO \& SILVA, 2004; GAMA et al., 2010; QUESADA et al., 2010). Estudos sobre o efeito da interação da correção da acidez do solo com a adubação fosfatada no crescimento de mudas de espécies nativas da Amazônia são escassos.

Diante do exposto, objetivou-se estimar o efeito da aplicação de doses de calcário e fósforo, assim como de suas interações, no desenvolvimento de mudas de tucumã cultivadas em amostras de solo com acidez elevada e baixo teor de $\mathrm{P}$ disponível.

\section{MATERIAL E MÉTODOS}

O experimento foi conduzido em viveiro de produção de mudas de tucumã da empresa Dendê do Tauá S/A (Dentauá), localizada no município de Santo Antônio do Tauá, Pará (latitude 0109' S, longitude 4807' O e 17 m de altitude).

Foram utilizadas amostras de um Argissolo Amarelo distrófico (SANTOS et al., 2013) coletadas em área da empresa Dentauá, na profundidade de $0-20 \mathrm{~cm}$. O solo foi analisado, de acordo com a metodologia descrita em SILVA (2009), e apresentou as seguintes características: $\mathrm{pH}\left(\mathrm{H}_{2} \mathrm{O}\right)=5,2 ; \mathrm{MO}=11,7 \mathrm{~g} \mathrm{~kg}^{-1} ; \mathrm{P}$ Mehlich-1 = $6 \mathrm{mg}$ $\mathrm{dm}^{-3} ; \mathrm{K}=0,06 \mathrm{cmo}_{\mathrm{c}} \mathrm{dm}^{-3} ; \mathrm{Ca}=0,6 \mathrm{cmol}_{\mathrm{c}} \mathrm{dm}^{-3} ; \mathrm{Mg}=0,3 \mathrm{cmol}_{\mathrm{c}} \mathrm{dm}^{-3} ; \mathrm{Al}=1,1 \mathrm{cmol}_{\mathrm{c}}$ $\mathrm{dm}^{-3} ; \mathrm{H}+\mathrm{Al}=6,4 \mathrm{cmol}_{\mathrm{c}} \mathrm{dm}^{-3} ; \mathrm{SB}=0,96 \mathrm{cmol}_{\mathrm{c}} \mathrm{dm}^{-3} ; \mathrm{CTC}=7,36 \mathrm{cmol}_{\mathrm{c}} \mathrm{dm}^{-3} ; \mathrm{V}=13$ $\% ; \mathrm{m}=53 \%$; Cu Mehlich-1 = 2,2 mg dm${ }^{-3}$; Mn Mehlich-1 = 4,0 mg dm ${ }^{-3}$; Fe Mehlich$1=252 \mathrm{mg} \mathrm{dm}$; Zn Mehlich-1 = 2,5 mg dm${ }^{-3}$; e $777 \mathrm{~g} \mathrm{~kg}^{-1}$ de areia, $143 \mathrm{~g} \mathrm{~kg}^{-1} \mathrm{de}$ silte e $80 \mathrm{~g} \mathrm{~kg}^{-1}$ de argila.

Os tratamentos foram distribuídos em delineamento inteiramente casualizado, em esquema fatorial $4 \times 6$, correspondendo a quatro níveis de saturação por bases $(13,40,65$ e $90 \%)$ e seis doses de $P\left(0,30,60,120,240\right.$ e $\left.420 \mathrm{mg} \mathrm{dm}^{-3}\right)$, com seis repetições, que totalizou 144 parcelas experimentais. Amostras de solo foram passadas em peneira de $4 \mathrm{~mm}$ de malha. Posteriormente, sacos plásticos de polietileno preto $(25 \mathrm{~cm} \times 30 \mathrm{~cm} \times 0,2 \mathrm{~mm})$, sanfonados e perfurados na base e na lateral, foram preenchidos com $3 \mathrm{dm}^{3}$ de solo.

O calcário utilizado apresentou PRNT de 91\%, 32\% de $\mathrm{CaO}$ e $15 \%$ de $\mathrm{MgO}$. A fonte de $\mathrm{P}$ aplicada foi o superfosfato triplo $\left(44 \%\right.$ de $\left.\mathrm{P}_{2} \mathrm{O}_{5}\right)$. O calcário foi aplicado e misturado a todo volume de solo. O superfosfato triplo foi aplicado ao solo 30 dias após a aplicação de calcário, incorporado ao solo a aproximadamente $10 \mathrm{~cm}$ de profundidade.

Realizou-se uma adubação complementar em todas as plantas, com aplicação de $200 \mathrm{mg} \mathrm{kg}^{-1}$ de $\mathrm{N}$ (metade dessa dose foi fornecida como ureia e o restante como sulfato de amônio) e $200 \mathrm{mg} \mathrm{kg}^{-1}$ de $\mathrm{K}$ fornecido como $\mathrm{KCl}$. Micronutrientes foram aplicados via solução nutritiva, nas seguintes doses: $0,5 \mathrm{mg} \mathrm{dm}^{-3}$ de $B ; 1,5 \mathrm{mg} \mathrm{dm}^{-3}$ de $\mathrm{Cu} ; 2,0 \mathrm{mg} \mathrm{dm}^{-3}$ de $\mathrm{Mn} ; 3,0 \mathrm{mg} \mathrm{dm}^{-3}$ de $\mathrm{Zn}$, utilizando como fontes $\mathrm{Na}_{2} \mathrm{~B}_{4} \mathrm{O}_{7} .10 \mathrm{H}_{2} \mathrm{O}, \mathrm{CuSO}_{4} .5 \mathrm{H}_{2} \mathrm{O}, \mathrm{MnSO}_{4} \cdot \mathrm{H}_{2} \mathrm{O}, \mathrm{ZnSO}_{4} \cdot \mathrm{H}_{2} \mathrm{O}$, respectivamente.

Após a emergência de plântulas em canteiro, foi realizado o transplante de plântulas para os sacos. As plantas foram cultivadas durante 350 dias, no período de agosto a julho. Após esse período, a parte aérea das plantas foi cortada rente ao 
solo e, em seguida, realizou-se a separação das raízes do solo. O material vegetal foi lavado com água destilada, acondicionado em sacos de papel e seco em estufa com circulação forçada de ar, à temperatura de $65^{\circ} \mathrm{C}$, até atingir massa constante. Posteriormente, determinou-se a massa de matéria seca da parte aérea (MSPA), de raiz (MSR) e total (MST = MSPA + MSR). Avaliaram-se também o diâmetro do coleto (DC), altura da planta, número de folhas lanceoladas por planta (NF) e o índice SPAD (clorofila).

Os dados foram submetidos à análise de variância, seguindo o modelo fatorial $4 \times 6$. Quando o $F$ foi significativo $(p<0,05)$, o efeito dos níveis de saturação por bases e das doses de $\mathrm{P}$ no desenvolvimento das mudas foi avaliado por meio de análise de regressão, considerando-se as regressões linear, quadrática e de superfície de resposta.

\section{RESULTADOS E DISCUSSÃO}

O resumo da análise de variância e os coeficientes de variação das variáveis avaliadas em mudas de tucumã, em função dos níveis de saturação por bases do solo e de doses de P, são apresentados na Tabela 1.

TABELA 1. Resumo da análise de variância para massa de matéria seca de parte área (MSPA), de raiz (MSR) e total (MST = MSPA + MSR), diâmetro do coleto, altura da planta, número de folhas por planta e índice SPAD.

\begin{tabular}{lcccc}
\hline \multirow{2}{*}{ Variável } & \multicolumn{2}{c}{ Fonte de variação } & \multicolumn{2}{c}{$\begin{array}{c}\text { Coeficiente de } \\
\text { variação (\%) }\end{array}$} \\
\cline { 2 - 4 } & $\begin{array}{c}\text { Saturação por bases } \\
(\mathrm{V})\end{array}$ & Fósforo $(\mathrm{P})$ & $\mathrm{V} \times \mathrm{P}$ & \\
\hline MSPA & $* *$ & $* *$ & $* *$ & 27,2 \\
MSR & $* *$ & $* *$ & $* *$ & 28,9 \\
MST & $* *$ & $* *$ & $* *$ & 23,2 \\
Diâmetro do coleto & $* *$ & $* *$ & $\mathrm{~ns}$ & 19,8 \\
Altura da planta & $\mathrm{ns}$ & $\mathrm{ns}$ & $\mathrm{ns}$ & 14,5 \\
Número de folhas & $* *$ & $* *$ & $\mathrm{~ns}$ & 20,9 \\
SPAD & $\mathrm{ns}$ & $\mathrm{ns}$ & $\mathrm{ns}$ & 17,4 \\
\hline
\end{tabular}

** significativo a $1 \%$ e ns: não significativo a $5 \%$.

Houve interação entre os fatores avaliados para a aplicação de doses de calcário e de $\mathrm{P}$ para massa de matéria seca da parte aérea, raiz e total de mudas de tucumã. GOMES et al. (2008) também observaram interação entre níveis de saturação por bases do solo e doses de $P$ para matéria seca da parte aérea e de raiz de mudas da espécie florestal garapa (Apuleia leiocarpa (Vogel) Macbride). Por outro lado, não se observou interação entre doses de calcário e de $\mathrm{P}$ para matéria seca da parte aérea, raiz e total em mudas de araçá-boi (Eugenia stipitata McVaugh) (MACEDO \& TEIXEIRA, 2012), biribazeiro (Rollinia mucosa (Jacq.) Bail) (TEIXEIRA et al., 2011) e mogno (Swietenia macrophylla King.) (CARDOSO et al., 2015).

A calagem promove vários efeitos em solos ácidos e, por isso, resulta em múltiplas interações com outros fatores de produção, com destaque para o aumento do aproveitamento do $\mathrm{P}$ nativo do solo ou de fertilizantes fosfatados solúveis em água (RAIJ, 2011). Neste estudo, observou-se que a aplicação de calcário influenciou a resposta do tucumã à aplicação de $\mathrm{P}$ (Tabela 1), pois as doses de $\mathrm{P}$ propiciaram o aumento da massa de matéria seca da parte aérea da muda e esse 
efeito foi dependente do nível de saturação por bases do solo (Figuras 1, 2 e 3). A redução da acidez do solo, por meio da calagem, é claramente relacionada com o potencial de resposta de plantas cultivadas à adubação fosfatada (SOUSA et al., 2007).

A calagem e a adubação fosfatada resultaram em efeito isolado no diâmetro do coleto e número de folhas por planta de mudas de tucumã. Também não houve interação também para a aplicação de calcário e $P$ para o crescimento do diâmetro do caule de mudas de araçá-boi (MACEDO \& TEIXEIRA, 2012) e de biribazeiro (TEIXEIRA et al., 2011).

Não houve efeito da correção da acidez do solo e da aplicação de $\mathrm{P}$ para a altura da planta e o índice SPAD. A não-significância da calagem e adubação fosfatada no índice SPAD indica que a correção da acidez e o suprimento de cálcio e magnésio (calcário) e fósforo (superfosfato triplo) não alteraram de modo expressivo o teor de clorofila das folhas avaliadas.

Os níveis de saturação por bases e doses de fósforo responsáveis pelos máximos valores de massa matéria seca da parte aérea, de raiz e total foram obtidos por equações de superfície de resposta apresentadas na Tabela 2.

TABELA 2. Superfície de resposta da massa de matéria seca de parte área (MSPA), de raiz (MSR) e total (MST = MSPA + MSR) em função de níveis de saturação por bases do solo $(\mathrm{V})$ e doses de fósforo $(\mathrm{P})$.

Variável Superfície de resposta

MSPA $Y=0,53+0,11 V+0,02 P-0,0008 V^{2}-0,00003 P^{2}+0,00004 V P\left(R^{2}=0,45\right)$ *

MSR $Y=1,97+0,05 V+0,01 P-0,0005 V^{2}-0,00003 P^{2}+0,0001 V P\left(R^{2}=0,43\right)$ **

MST $Y=2,51+0,16 V+0,03 P-0,0013 V^{2}-0,00006 P^{2}+0,00014 V P\left(R^{2}=0,48\right)$ **

Y: massa de matéria seca de parte área, de raiz ou total (g/planta). ${ }^{* \star}$ significativo a $1 \%$

Os valores relativamente baixos de $R^{2}$ das equações de superfície de resposta (Tabela 2) podem ser explicados pela ausência de efeito em todos os níveis de saturação por bases e doses de $P$ avaliados (Figuras 1, 2 e 3). A massa de matéria seca de parte aérea, raiz e total não foi influenciada tanto nas doses mais baixas de calcário ( $\mathrm{V}=13$ ou $40 \%)$ como de $\mathrm{P}\left(0\right.$ ou $\left.30 \mathrm{mg} \mathrm{dm}^{-3}\right)$, provavelmente por causa da fixação de $\mathrm{P}$ em condições de solo ácido e limitação de $\mathrm{P}$ disponível no substrato para o crescimento das plantas.

A MSPA aumentou de forma quadrática em resposta aos níveis crescentes de $V$, nas doses de $P \geq$ a $60 \mathrm{mg} \mathrm{dm}^{-3}$ (Figura $1 \mathrm{~A}$ ), ou seja, não houve resposta a aplicação de calcário em dose baixa de $\mathrm{P}\left(30 \mathrm{mg} \mathrm{dm}^{-3}\right)$ ou na ausência de adubação fosfatada. O máximo valor de MSPA (6,6 g/planta) foi obtido no nível de saturação por bases de $73 \%$, conforme equação de superfície de resposta (Tabela 2), e correspondeu a um aumento de 2,4 vezes comparado ao tratamento sem aplicação de calcário.

O acúmulo de MSPA também apresentou resposta quadrática à aplicação de doses de calcário em mudas de araçá-boi, quando cultivado em um Latossolo muito argiloso da região Amazônica (MACEDO \& TEIXEIRA, 2012). GOMES et al. (2008) verificaram que a dose de calcário necessária para atingir $90 \%$ da máxima produção de MSPA de mudas de garapa, que é uma espécie arbórea de ocorrência no Estado do Pará, foi aquela suficiente para elevar $\mathrm{V}$ a $60 \%$. Embora essas espécies sejam ENCICLOPÉDIA BIOSFERA, Centro Científico Conhecer - Goiânia, v.13 n.23; p.1185 
geralmente originárias de locais onde predominam solos ácidos, todas responderam positivamente a correção da acidez, evidenciando, portanto, o potencial de resposta de espécies da Amazônia à aplicação de calcário. Historicamente, a palma de óleo (Elaeis guineensis Jacq.), que pertence à mesma família do tucumã (Arecaceae), foi considerada tolerante a solos ácidos (AUXTERO \& SHAMSHUDDIN, 1991), no entanto verificou-se mais recentemente que esta cultura é favorecida pela correção da acidez do solo (CRISTANCHO et al., 2011).

Houve efeito quadrático para a produção de MSPA de mudas de tucumã em resposta a aplicação de doses de $\mathrm{P}$, apenas nos níveis de $\mathrm{V}$ de 65 e 90\% (Figura 1B). A máxima produção de MSPA (6,6 g/planta) foi atingida na dose de $P$ de 260 $\mathrm{mg} \mathrm{dm}^{-3}$ (Tabela 2) e foi 2,3 vezes maior que a obtida no tratamento sem aplicação de $\mathrm{P}$. Na ausência de aplicação de calcário e na $\mathrm{V}$ de $40 \%$, a adubação fosfatada não influenciou a MSPA (Figura 1B).
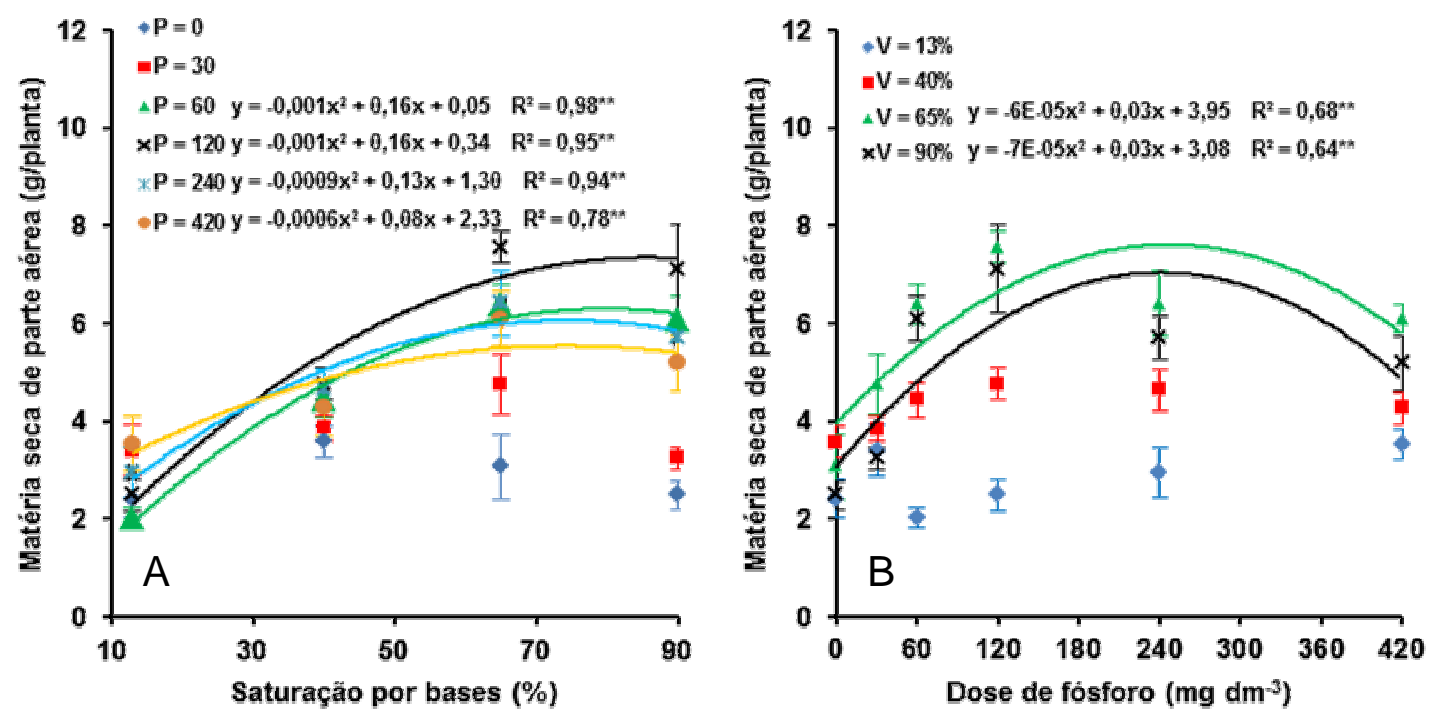

FIGURA 1. Massa de matéria seca de parte aérea de mudas de tucumã em função de níveis crescentes de saturação por bases do solo (A) e doses de fósforo (B).

GOMES et al. (2008) observaram que as doses de $\mathrm{P}$ necessárias para a obtenção de $90 \%$ da máxima produção de MSPA variou de 54 a $191 \mathrm{mg} \mathrm{dm}^{-3}$, respectivamente para $V$ de 60 e $40 \%$. Na presença de calcário, em dose suficiente para elevar a $\mathrm{V}$ a $60 \%$, observou-se que a máxima produção de MSPA de vetiver (Chrysopogon zizanioides (L.) Roberty syn. Vetiveria zizanioides (L.) Nash), cultivado em amostras de solos do estado do Amazonas, foi atingida na dose de $\mathrm{P}$ de aproximadamente $250 \mathrm{mg} \mathrm{dm}^{-3}$; no entanto, sem aplicação de calcário, seria necessário dose de $\mathrm{P}$ superior a $400 \mathrm{mg} \mathrm{dm}^{-3}$ para obter-se o mesmo resultado (TEIXEIRA et al., 2015). Em mudas de araçá-boi, a maior produção de MSPA foi obtida com a aplicação de dose de $\mathrm{P}$ muito superior à encontrada em neste estudo (cerca de $560 \mathrm{mg} \mathrm{dm}^{-3}$ ), possivelmente porque foi utilizado um solo muito argiloso como substrato (MACEDO \& TEIXEIRA, 2012).

Nos tratamentos sem aplicação ou na dose de $30 \mathrm{mg} \mathrm{dm}^{-3}$ de $P$, os níveis crescentes de V não influenciaram a MSR (Figura 2A). No entanto, nos tratamentos 
com doses de $\mathrm{P}$ superiores a $60 \mathrm{mg} \mathrm{dm}^{-3}$, houve aumento quadrático das doses de calcário na produção de MSR. A saturação por bases do solo de aproximadamente $85 \%$ resultou em máxima produção de MSR (6,5 g/planta). Neste nível de V, a MSR foi muito superior (aproximadamente duas vezes) à obtida nos tratamentos sem aplicação de calcário.

Cultivado em amostras da camada superficial de um Latossolo Amarelo distrófico proveniente do Amazonas, a máxima produção de MSR de mudas de biribazeiro foi obtida em dose de calcário de $4,4 \mathrm{t} \mathrm{ha}^{-1}$, que resultou em incremento de $45 \%$ em relação ao tratamento controle (TEIXEIRA et al., 2011). MACEDO \& TEIXEIRA (2012) também observaram aumento na MSR de mudas de araçá-boi em resposta à calagem, quando cultivado em um Latossolo Amarelo do estado do Amazonas. Esses resultados destacam a importância da correção da acidez do solo, com neutralização de $\mathrm{Al}^{3+}$ tóxico e fornecimento de nutrientes, especialmente o $\mathrm{Ca}$, para o desenvolvimento vigoroso do sistema radicular de espécies da região Amazônica.

Nos tratamentos sem aplicação ou em baixa dose de calcário ( $V=40 \%$ ) não houve efeito da aplicação de $P$ na MSR (Figura 2B). Similarmente ao observado para a MSPA, as doses de $P$ aumentaram quadraticamente a MSR, somente nos níveis de $\mathrm{V}$ de 65 e $90 \%$. A máxima produção de MSR (6,5 g/planta) foi obtida na dose de $P$ de $340 \mathrm{mg} \mathrm{dm}^{-3}$ (Tabela 2) e correspondeu a um aumento de 2,5 vezes em relação aos tratamentos sem aplicação de $P$.

$\mathrm{Na}$ presença de calagem $(\mathrm{V}=60 \%)$, TEIXEIRA et al. (2015) verificaram a necessidade de aplicação de aproximadamente $250 \mathrm{mg} \mathrm{dm}^{-3}$ de $\mathrm{P}$ para obter-se a máxima produção de MSR de vetiver. Os resultados deste estudo mostram que o efeito positivo da adubação fosfatada no crescimento do sistema radicular da muda de tucumã foi dependente da correção da acidez do solo, o que confirma também para essa espécie vegetal amazônica a sinergia amplamente conhecida entre a calagem e o uso eficiente de fertilizante fosfatado para plantas tradicionalmente cultivadas.
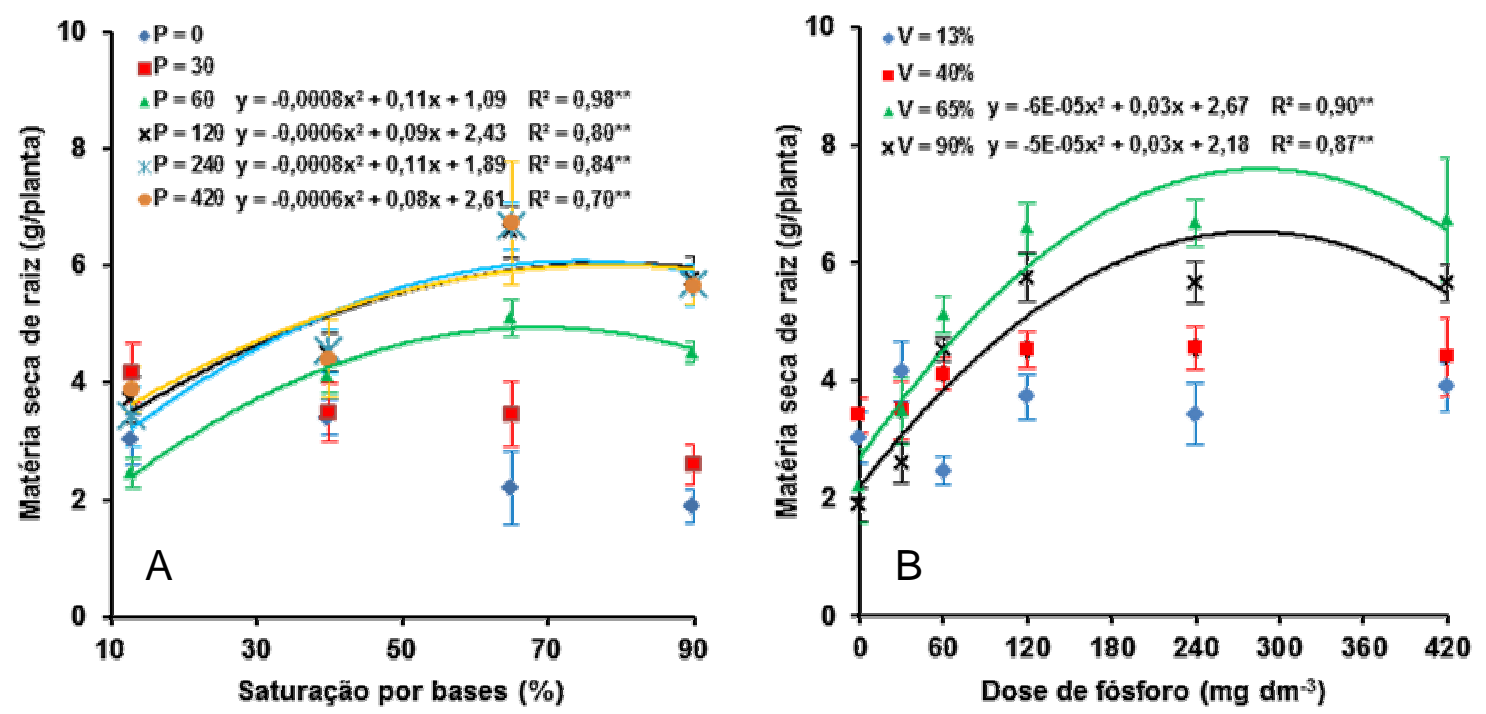

FIGURA 2. Massa de matéria seca de raiz de mudas de tucumã em função de níveis crescentes de saturação por bases do solo $(A)$ e doses de fósforo $(B)$.

Os níveis crescentes de V influenciaram a MST de mudas de tucumã (Figura 3A) de maneira similar aos resultados observados na MSPA (Figura 1A) e MSR 
(Figura 2A). As doses de calcário resultaram em aumentos quadráticos na MST nos tratamentos em que o $\mathrm{P}$ foi fornecido as plantas em doses superiores a $60 \mathrm{mg} \mathrm{dm}^{-3}$. Portanto, não houve resposta à calagem na ausência de adubação fosfatada ou quando foi aplicada a dose de $30 \mathrm{mg} \mathrm{dm}^{-3}$ de $\mathrm{P}$, indicando que o potencial de resposta em desenvolvimento das plantas à calagem depende da presença de $\mathrm{P}$ no substrato e em quantidade suficiente.

O máximo valor de MST (13 g/planta) foi obtido em $V$ de aproximadamente $75 \%$ (Tabela 2) e correspondeu a um aumento de cerca de duas vezes em comparação aos tratamentos sem aplicação de calcário. TEIXEIRA et al. (2011) observaram que a produção de MST de mudas de biribazeiro, cultivado em um Latossolo Amarelo distrófico do Amazonas, foi obtida com a aplicação de 5,5 t ha ${ }^{-1}$ de calcário e resultou em incremento de $40 \%$ em relação ao tratamento sem calagem. Em um Latossolo Amarelo distrófico do Amazonas, a calagem também aumentou a produção de MST de mudas de araçá-boi, sendo necessária a aplicação de $3,5 \mathrm{~g}$ de calcário por muda para obter-se a máxima produção (MACEDO \& TEIXEIRA, 2012).

Nos níveis de $\mathrm{V}$ de 65 e $90 \%$, as doses de $\mathrm{P}$ também aumentaram a MST de mudas de tucumã, ambos com ajuste quadrático (Figura 3B). Por outro lado, as doses de $\mathrm{P}$ não influenciaram a MST na ausência de correção da acidez do solo ou na $V$ de $40 \%$, possivelmente em função da fixação de $\mathrm{P}$ em condições de solo ácido. A máxima produção de MST (13 g/planta) foi atingida na dose de $300 \mathrm{mg} \mathrm{dm}^{-3}$ de $P$ (Tabela 2) e foi muito superior (aumento de 2,4 vezes) à obtida nos tratamentos sem aplicação de $P$.

O máximo valor de MST de vetiver foi atingido em dose de $250 \mathrm{mg} \mathrm{dm}^{-3}$ de $\mathrm{P}$ quando aplicou-se calcário suficiente para aumentar a $\mathrm{V}$ a $60 \%$, no entanto, na ausência de correção da acidez do solo, seria necessário doses de $P$ superior a 400 $\mathrm{mg} \mathrm{dm}^{-3}$ para obter-se o mesmo efeito (TEIXEIRA et al., 2015). MACEDO \& TEIXEIRA (2012) observaram máxima produção de MST de mudas de araçá-boi com a aplicação de $545 \mathrm{mg} \mathrm{kg}^{-1}$ de P. Esta dose foi muito superior a dose observada neste estudo, provavelmente devido ao alto teor de argila (71\%) do solo utilizado como substrato, comparado ao usado no tucumã ( $8 \%$ de argila).
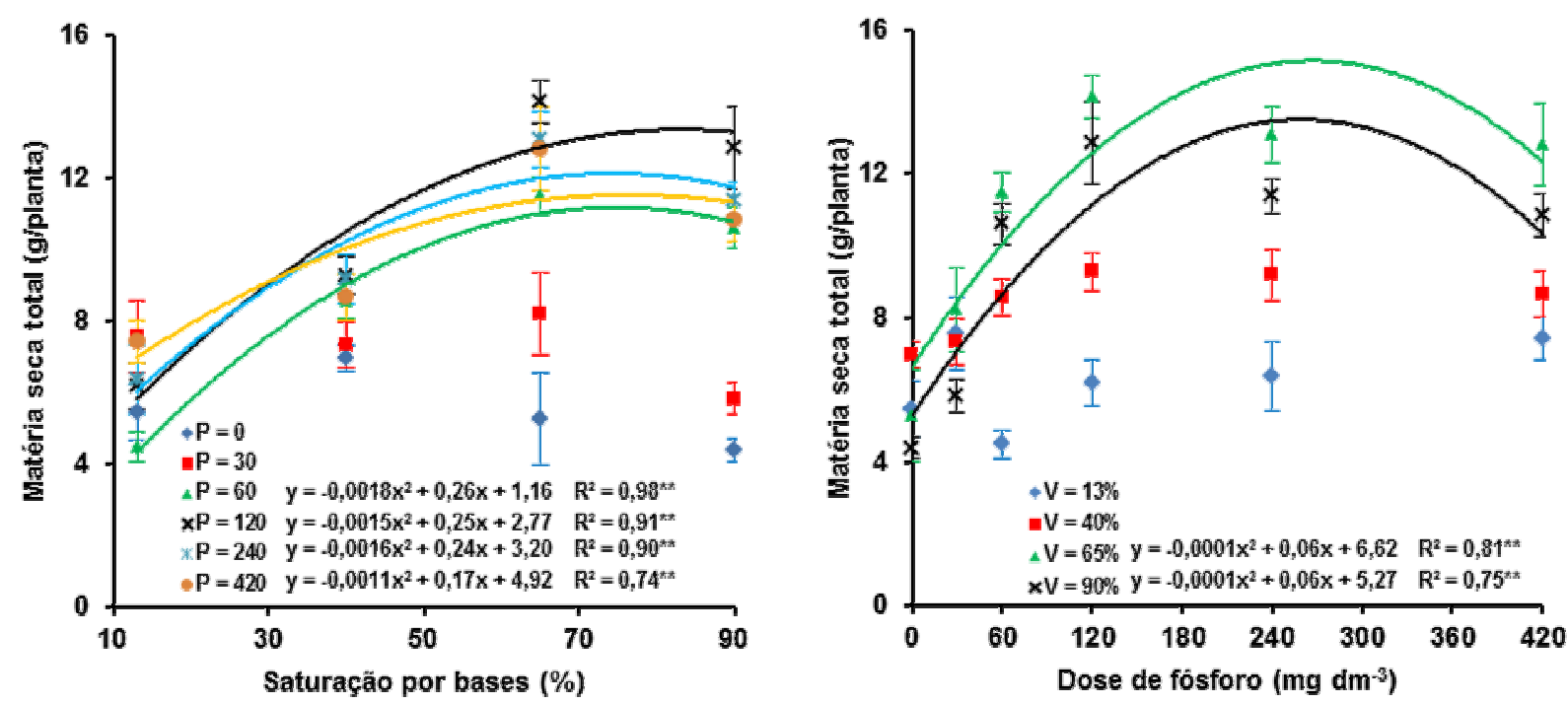

FIGURA 3. Massa de matéria seca total (parte aérea + raiz) de mudas de tucumã em função de níveis crescentes de saturação por bases do solo e doses de fósforo. 
Os níveis crescentes de $\mathrm{V}$ do solo aumentaram o diâmetro do coleto (DC) de mudas de tucumã de forma quadrática (Figura 4A). O maior DC da planta observado foi de $10 \mathrm{~mm}$, que foi 1,5 vezes maior que o DC obtido no tratamento sem aplicação de calcário, e foi alcançado na $\mathrm{V}$ de aproximadamente $75 \%$.

O máximo crescimento no diâmetro do colo de mudas de biribazeiro representou um aumento de $20 \%$ em comparação ao tratamento controle e foi alcançado com a aplicação de dose equivalente a $4,4 \mathrm{t} \mathrm{ha}^{-1}$ de calcário (TEIXEIRA et al., 2011). Observou-se que outros produtos, além do calcário, como escória de siderurgia e óxido de magnésio, apresentam potencial de correção da acidez do solo e de aumentar o diâmetro do caule de mudas de cafeeiro (MARTINS et al., 2010).

Os valores de diâmetro do coleto também se ajustaram ao modelo quadrático em resposta às doses de $\mathrm{P}$ aplicadas (Figura 4B). O máximo valor de DC obtido também foi de $10 \mathrm{~mm}$, que foi equivalente a 1,3 vezes ao $\mathrm{DC}$ medido no tratamento sem adubação fosfatada, e foi atingido na dose de aproximadamente $300 \mathrm{mg} \mathrm{dm}^{-3}$ de P. MACEDO \& TEIXEIRA (2012) observaram máximo diâmetro do coleto de mudas de araçá-boi em doses equivalentes a aproximadamente $3,6 \mathrm{t} \mathrm{ha}^{-1}$ de calcário e $470 \mathrm{mg} \mathrm{kg}^{-1}$ de P. O máximo diâmetro do caule de maracujazeiro, cultivado em um Latossolo Amarelo distrófico do estado do Pará ( $9 \%$ de argila), em foi obtido com a saturação por bases de $77 \%$ e dose de $P$ de $249 \mathrm{mg} \mathrm{dm}^{-3}$ (BRASIL \& NASCIMENTO, 2010).
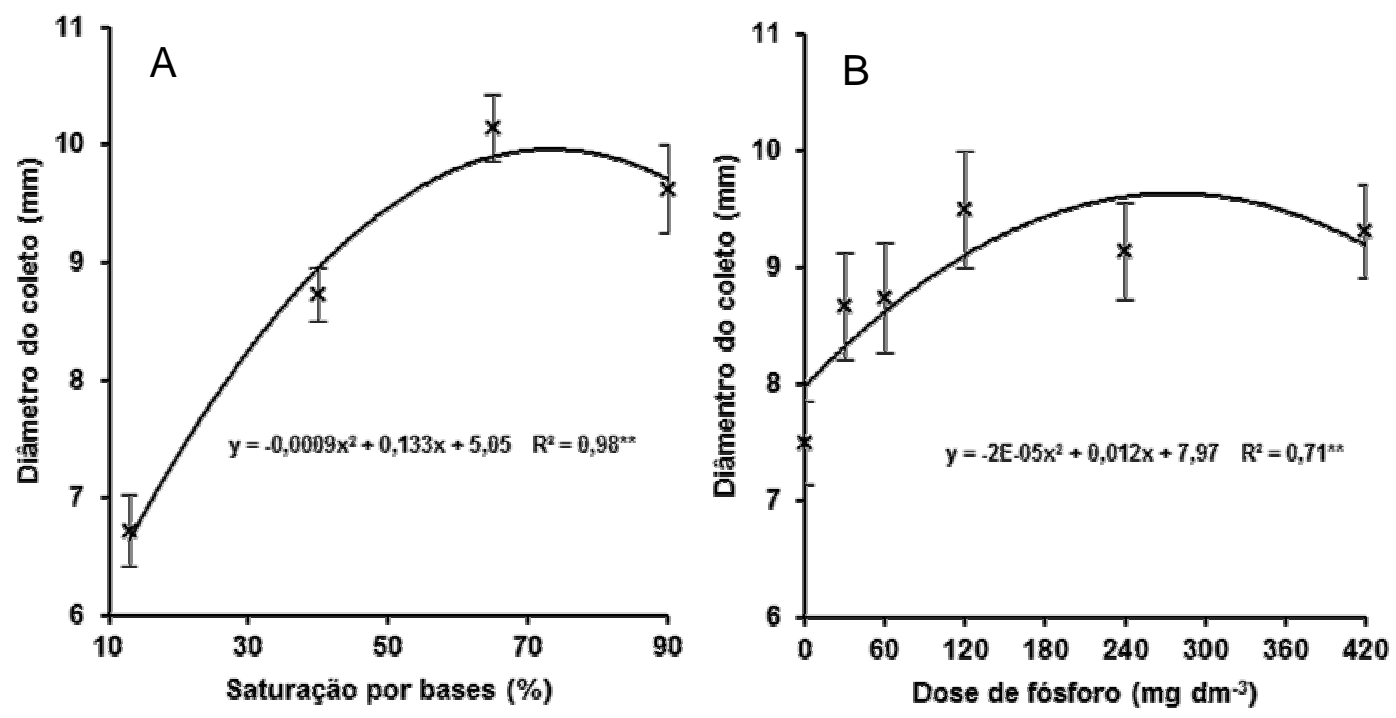

FIGURA 4. Diâmetro do coleto de mudas de tucumã em função de níveis de saturação por bases do solo (A) e doses de fósforo (B).

Houve aumento do número de folhas lanceoladas por planta em resposta aos níveis crescentes de $\mathrm{V}$, com ajuste quadrático (Figura 5A). O maior NF foi obtido na $\mathrm{V}$ de cerca de $70 \%$ e correspondeu ao aumento de $20 \%$ em comparação ao tratamento controle, sem aplicação de calcário. As doses de $\mathrm{P}$ também aumentaram de maneira quadrática $\mathrm{o} \mathrm{NF}$, sendo que o máximo $\mathrm{NF}$, obtido na dose de aproximadamente $250 \mathrm{mg} \mathrm{dm}^{-3}$ de $\mathrm{P}$, foi $30 \%$ superior ao do tratamento sem adição de $\mathrm{P}$

O número de folhas de milho e de mudas de maracujá também aumentou em resposta à correção da acidez do solo (ANDREOTTI, 2001; SOUZA, 2009). As doses de $\mathrm{P}$ também aumentaram o número de folhas de rabanete (OLIVEIRA, ENCICLOPÉDIA BIOSFERA, Centro Científico Conhecer - Goiânia, v.13 n.23; p.1189 2016 
2010), mas não o número de folhas expandidas de capim-tanzânia (PATÊS et al., 2007).


FIGURA 5. Número de folhas de mudas de tucumã em função de níveis de saturação por bases do solo (A) e doses de fósforo (B).

Os níveis de $\mathrm{V}$ relativamente elevados para se atingir o ponto de máximo das variáveis avaliadas no presente estudo evidenciam o potencial de resposta de mudas de tucumã à calagem. Além disso, é possível que as doses de calcário aplicadas no solo não tenham sido suficientes para se alcançar a $\mathrm{V}$ desejada teórica, mas o solo não foi analisado após a aplicação dos tratamentos. Nesse sentido, seriam recomendados mais estudos de curvas de neutralização da acidez do solo e de relação entre doses de corretivos e saturação por bases em solos representativos do estado do Pará. Como observado em outros trabalhos, que avaliaram a formação de mudas de espécies nativas da Amazônia, verificou-se que a calagem e a adubação fosfatada também podem contribuir para a obtenção de mudas de tucumã mais vigorosas.

\section{CONCLUSÃO}

A aplicação de calcário e fertilizante fosfatado melhora o desenvolvimento de mudas de tucumã quando cultivadas em um solo ácido e com baixo teor de fósforo disponível.

\section{AGRADECIMENTOS}

À Financiadora de Estudos e Projetos (Finep) pelo apoio financeiro por meio do projeto Propalma ( $\mathrm{n}^{\circ}$ 0487/08). Ao CNPq, por conceder bolsa de iniciação científica ao terceiro autor. À Empresa Dendê do Tauá S/A (Dentauá) pelo fornecimento de estrutura física e apoio operacional na condução do experimento.

\section{REFERÊNCIAS}

ANDREOTTI, M; SOUZA, E. C. A. de; CRUSCIOL, C. A. C. Componentes morfológicos e produção de matéria seca de milho em função da aplicação de calcário e zinco. Scientia Agricola, v. 58, n. 2, p.321-327, 2001. Disponível em: < 
https://dx.doi.org/10.1590/S0103-90162001000200015>. doi: 10.1590/S010390162001000200015

ATABANI, A. E.; SILITONGA, A. S.; ONG, H. C.; MAHLIA, T. M. I.; MASJUKI, H. H.; BADRUDDIN, I. A.; FAYAZ, H. Non-edible vegetable oils: A critical evaluation of oil extraction, fatty acid compositions, biodiesel production, characteristics, engine performance and emissions production. Renewable \& Sustainable Energy Reviews, v. 18, n. 1, p. 211-245, 2013. Disponível em: < https://dx.doi.org/10.1016/j.rser.2012.10.013>. doi: 10.1016/j.rser.2012.10.013

AUXTERO, E. A.; SHAMSHUDDIN, J. Growth of oil palm (Elaeis guineensis) seedlings on acid sulfate soils as affected by water regime and aluminum. Plant and Soil, v. 137, n. 2, p. 243-257, 1991. Disponível em: <http://dx.doi.org/ 10.1007/BF00011203>. doi: 10.1007/BF00011203

BRASIL, E. C.; NASCIMENTO, E. V. S. do. Influência de calcário e fósforo no desenvolvimento e produção de variedades de maracujazeiro-amarelo. Revista Brasileira de Fruticultura, v. 32, n. 3, p. 892-902, 2010. Disponível em: <http://dx.doi.org/10.1590/S0100-29452010005000092>. doi: 10.1590/S010029452010005000092

CARDOSO, A. A. S.; SANTOS, J. Z. L.; TUCCI, C. A. F.; FARIAS, E. P.; MOURA, R. P. M. Influência da acidez e do teor de fósforo do solo no crescimento inicial do mogno. Brazilian Journal of Forestry Research, v. 35, n. 81, p. 7, 2015. Disponível em: < $\quad$ http://dx.doi.org/10.4336/2015.pfb.35.81.667>. doi: 10.4336/2015.pfb.35.81.667.

CORLEY, R. H. V. How much palm oil do we need? Environmental Science \& Policy, v. 12, n.2, p. 134-139, 2009. Disponível em: < http://dx.doi.org/ 10.1016/j.envsci.2008.10.011>. doi: 10.1016/j.envsci.2008.10.011

CRISTANCHO, J. A. R.; HANAFI, M. M.; OMAR, S. R. S.; RAFII, Y. M.; MARTÍNEZ, F. M.; CAMPOS, C. E. C. Alleviation of aluminum in acidic soils and its effect on growth of hybrid and clonal oil palm seedlings. Journal of Plant Nutrition, v. 34, n. 3, p. 387-401, 2011. Disponível em: < http://dx.doi.org/ 10.1080/01904167.2011.536880>. doi: 10.1080/01904167.2011.536880

FAGERIA, N. K.; NASCENTE, A. S. Management of Soil Acidity of South American Soils for Sustainable Crop Production. Advances in Agronomy, v.128, p.221-275, 2014.Disponível em: < http://dx.doi.org/10.1016/B978-0-12-802139-2.00006-8>. doi: 10.1016/B978-0-12-802139-2.00006-8

FALCÃO, N. P. S.; SILVA, J. R. A. Características de adsorção de fósforo em alguns solos da Amazônia Central. Acta Amazonica, v. 34, n.3, p. 337-342, 2004. Disponível em: <http://dx.doi.org/10.1590/S0044-59672004000300001>. doi: 10.1590/S0044-59672004000300001

FOOD AND AGRICULTURE ORGANIZATION OF THE UNITED NATIONS - FAO [1995]. Crops.1995 Disponível em: <http://faostat.fao.org>. 
GAMA, J. R. N. F.; CARVALHO, E. J. M.; RODRIGUES, T. E.; VALENTE, M. A. Solos do Estado do Pará. In: CRAVO, M. S.; VIÉGAS, I. J. M.; BRASIL, E. C. Recomendação de Adubação e Calagem para o Estado do Pará, $1^{a}$ ed. rev. atual., Belém, PA: Embrapa Amazônia Oriental, p. 19-30, 2010.

GOMES, K. C. O.; PAIVA, H. N.; NEVES, J. C. L.; BARROS, N. F.; SILVA, R. S. Crescimento de mudas de garapa em resposta à calagem e ao fósforo. Revista Arvore, v. 22, n. 3, p. 387-394, 2008. Disponível em: <http://dx.doi.org/10.1590/S0100-67622008000300001>. doi: 10.1590/S010067622008000300001

$\mathrm{KOH}$, L. P. Potential habitat and biodiversity losses from intensified biodiesel feedstock production. Conservation Biology, v. 21, n. 5, p. 1373-1375. 2007. Disponível em: <http://dx.doi.org/10.1111/j.1523-1739.2007.00771.x>. doi: 10.1111/j.1523-1739.2007.00771.x

MACEDO, S. T.; TEIXEIRA, P. C. Calagem e adubação fosfatada para formação de mudas de araçá-boi. Acta Amazonica, v. 32, n.3, p.407-409, 2012. Disponível em:< http://dx.doi.org/10.1590/S0044-59672012000300013>. doi: 10.1590/S004459672012000300013

MARTINS, I. D.; TOMAZ, M. A.; BRINATE, S. V. B.; NOGUEIRA, N. O.; CANDIDO, A. O. Comparação das doses de corretivos de acidez do solo utilizando variáveis de crescimento do café arábica como indicador em dois latossolos. Enciclopédia Biosfera, v. 6, n.10, p. 4-7, 2010.

NASCIMENTO, W. M. O.; OLIVEIRA M. S. P. Produção de Mudas de Tucumanzeiro-do-pará (Astrocaryum vulgare Mart.) por Perfilhos. Belém: Embrapa Amazônia Oriental, 5p. (Embrapa Amazonia Oriental, Comunicado Técnico, 230), 2011.

]

NATALE, W.; PRADO, R. M.; LEAL, R. M.; FRANCO, C. F. Efeitos da aplicação de zinco no desenvolvimento, no estado nutricional e na produção de matéria seca de mudas de maracujazeiro. Revista Brasileira de Fruticultura, v. 26, n. 2, p. 310-314, 2004. Disponível em: <http://dx.doi.org/10.1590/S0100-29452004000200031>. doi: 10.1590/S0100-29452004000200031

NO, SY. Inedible vegetable oils and their derivatives for alternative diesel fuels in $\mathrm{Cl}$ engines: a review. Renewable \& Sustainable Energy Reviews, v. 15, n. 1, p.13149, 2011. Disponível em: <http://dx.doi.org/10.1016/j.rser.2010.08.012>. doi: 10.1016/j.rser.2010.08.012

OLIVEIRA, A. S.; NETO, A. J. S.; RIBEIRO, A.; RASCON, N. J. L.; RODY, Y. P.; ALMEIDA, A. Q. Determinação do tempo térmico para o desenvolvimento de mudas de eucalipto na fase de enraizamento. Revista Brasileira de Engenharia Agrícola e Ambiental, v. 16, n. 11, p. 1223-1228, 2012. Disponível em: <http://dx.doi.org/10.1590/S1415-43662012001100011>. doi: 10.1590/S141543662012001100011 
OLIVEIRA, F R A de; OLIVEIRA, F A de, MEDEIROS, J F de; SOUSA, V F L de; Freire, A G. Interação entre salinidade e fósforo na cultura do rabanete. Revista Ciência Agronômica, v. 41, n. 4, p. 519-526, 2010. Disponível em: <http://dx.doi.org/10.1590/S1806-66902010000400003>. doi: 10.1590/S180666902010000400003

OLIVEIRA, R. P.; NINO, A. F. P; NICKEL, O. Limpeza de patógenos e propagação in vitro de cultivares de pereira. Pelotas: Embrapa Clima Temperado, 2004. (Embrapa Clima Temperado. Comunicado técnico, 104).

OSAKI, M.; BATALHA, M. O. Produção de biodiesel e óleo vegetal no Brasil: realidade e desafio. Organizações Rurais \& Agroindustriais, v. 13, n. 2, p. 227 242, 2011.

PATÊS, N. M. S.; PIRES, A. J. V.; SILVA, C. C. F. da; SANTOS, L. C.; CARVALHO, G. G. P. de; FREIRE, M. A. L. Características morfogênicas e estruturais do capimtanzânia submetido a doses de fósforo e nitrogênio. Revista Brasileira de Zootecnia, v. 36, n. 6, p. 1736-1741, 2007. Disponível em: <http://dx.doi.org/10.1590/S1516-35982007000800005>. doi: 10.1590/S151635982007000800005

QUESADA, C. A.; LLOYD, J., SCHWARZ, M.; PATIÑO, S.; BAKER, T. R.; CZIMCZIK, C.; FYLLAS, N. M.; MARTINELLI, L.; NARDOTO, G. B.; SCHMERLER, J.; SANTOS, A. J. B.; HODNETT,M. G.; HERRERA, R.; LUIZÃO, F. J.; ARNETH, A.; LLOYD, G.; DEZZEO, N.; HILKE, I.; KUHLMANN, I.; RAESSLER, M.; BRAND, W. A.; GEILMANN, H.; MORAES FILHO, J.O.; CARVALHO, F.P.; FILHO, A. R. N.; CHAVES, J. E.; CRUZ JUNIOR, O. F.; PIMENTEL, T. P.; PAIVA, R. Variations in chemical and physical properties of Amazon forest soils in relation to their genesis. Biogeosciences, v. 7, n. 5, p. 1515-1541, 2010. Disponível em: < http://dx.doi.org/10.5194/bg-7-1515-2010>. doi: 10.5194/bg-7-1515-2010

RAIJ, B. van. Fertilidade do solo e manejo de nutrientes. Piracicaba: International Plant Nutrition Institute, 2011. 420p.

RIVAL, A.; LEVANG, P. Palms of controversies: Oil palm and development challenges. Bogor, Indonesia: CIFOR, 2014. 60p.

SANCHEZ, P. A.; COCHRANE, T. T. Soils constraints in relation to major farming systems of tropical America. International Rice Research Institute and New York state college of agriculture and life sciences, Cornel University, Los Baños. p. 106-139, 1980.

SANTOS, H.G. dos; JACOMINE, P.K.T.; ANJOS, L.H.C. dos; OLIVEIRA, V.A. de; LUMBRERAS, J.F.; COELHO, M.R.; ALMEIDA, J.A. de; CUNHA, T.J.F.; OLIVEIRA, J.B. de. Sistema brasileiro de classificação de solos. 3.ed. Brasília: Embrapa, 2013. 353p.

SHANLEY, P.; SERRA, M.; MEDINA, G. Frutíferas e Plantas Úteis na Vida Amazônica. 2 ${ }^{\underline{a}}$ ed. Belém: CIFOR, Imazon, 316p. 2010. 
SILVA, E. M.; CARVALHO, G. R.; ROMANIELLO, M. M. Mudas de cafeeiros: tecnologia de produção. Belo Horizonte: EPAMIG, 56p., 2000. (EPAMIG, Boletim Técnico, 60).

SILVA, F. C. Manual de análises químicas de solos, plantas e fertilizantes. 2.ed. Brasília, Embrapa Informações Tecnológicas, 2009. 627p.

SOUZA, D. M. G.; MIRANDA, L. N.; OLIVEIRA, S. A. Acidez do solo e sua correção. In: NOVAIS, R. F.; ALVAREZ V., V. H.; BARROS, N. F.; FONTES, R. L. F.; CANTARUTTI, R. B.; NEVES, J. C. L., eds. Fertilidade do solo. Viçosa, MG, Sociedade Brasileira de Ciência do Solo, 2007. p.205-274.

SOUZA, H. A. de, RAMOS, J. D., MELO, P. C. de, HAFLE, O. M., RODRIGUES, H. C. A; Santos, V. A. dos. Avaliação de doses e produtos corretores da acidez em variáveis biométricas na produção de mudas de maracujazeiro. Acta Scientiarum. Agronomy, v. 31, n. 4, p. 607-612, 2009. Disponível em: < http://dx.doi.org/10.4025/actasciagron.v31i4.529> doi: 10.4025/actasciagron.v31i4.529

TEIXEIRA, P. C.; MACEDO, S. T. Calagem e fósforo para a formação de mudas de biribazeiro. Revista de Ciências Agrárias, Amazonian journal of agricultural and environmental sciences, v. 42, n. 3, p. 262-264, 2011. Disponível em: < http://dx.doi.org/10.4322/rca.2012.021>. doi:10.4322/rca.2012.021

TEIXEIRA, P. C.; MESQUITA, I.L.; MACEDO, S. T.; TEIXEIRA, W. G.; LIMA, W. A. A. Resposta de vetiver à aplicação de calcário e fósforo em três classes de solo. Revista Brasileira de Engenharia Agricola e Ambiental, v. 19, n. 2, p. 99-105, 2015. Disponível em: <http://dx.doi.org/10.1590/1807-1929/agriambi.v19n2p99-105>. doi: 10.1590/1807-1929/agriambi.v19n2p99-105

TUCCI, C. A. F.; LIMA, H. N.; LESSA, J. F. Adubação nitrogenada na produção de mudas de mogno (Swietenia macrophylla King). Acta Amazonica, v. 39, n. 2, p. 289-294, 2009. Disponível: <http://dx.doi.org/10.1590/S0044-59672009000200007>. doi: 10.1590/S0044-59672009000200007

UNITED STATES DEPARTAMENT OF AGRICULTURE. Foreign Agriculture Service. Production, supply and distribution online. 2016 Disponível em: $<$ http://apps.fas.usda.gov/psdonline>.

VALENCIA, W. H.; SAMPAIO, P. T. B.; SOUZA, L. A. G. Crecimiento inicial de Palo de Rosa (Aniba rosaeodora Ducke) en distintos ambientes de fertilidad. Acta Amazonica, v. 40, n. 4, p. 693-698, 2010. Disponível em: <http: //dx.doi.org/10.1590/S0044-59672010000400008 >. doi:10.1590/S004459672010000400008 